\title{
Transforming scientific evidence into better consumer choices
}

\author{
Janet G Bauer $^{1} *$ \& Francesco Chiappelli $^{1}$
}

${ }^{1}$ Division of Restorative Dentistry, June and Paul Ehrlich Endowed Program in Geriatric Dentistry, 23-008E CHS, UCLA School of Dentistry, PO Box 951668, 10833 Le Conte Avenue, Los Angeles, California 90095-1668; Janet G Bauer- Email: jbauer@dent.ucla.edu; Phone: 310-825-57747; Fax: 310641-0523; *Corresponding author

Received December 15, 2010; Accepted December 21, 2010; Published January 06, 2011

\begin{abstract}
:
Translational research using evidence-based and comparative effectiveness research continues to evolve, becoming a useful tool in improving informed consent and decision-making in the clinical setting. While in development, emerging technologies, including cellular and molecular biology, are leading to establishing evidence-based dental practices. One emerging technology, which conjoins bench proteomic findings to clinical decision-making for treatment intervention, is the Translational Evidence Mechanism. This mechanism was developed to be a foundation for a compact between researcher, translational researcher, clinician, and patient. The output of such a mechanism is the clinical practice guideline (CPG), an interactive tool for dentists and patients to game evidence in reaching optimum clinical decisions that correspond to individual patient preferences and values. As such, the clinical practice guideline requires the vesting of decision, utility, and cost best evidence. Evidence-based research provides decision data, a first attempt at supporting decision-making by providing best outcome data. Since then comparative effectiveness research has emerged, using systematic review analysis to compare similar treatments or procedures in maximizing the choice of the most effective cost/benefit option within the context of best evidence. With innovation in the clinical practice guideline for optimizing efficacy and comparative effectiveness research, evidence-based practices will shape a new approach to health-based systems that adhere to shared decision-making between bench scientists, healthcare providers and patients.
\end{abstract}

Keywords: Translational evidence, clinical decision-making, clinical practice guidelines (CPG's), evidence-based practice, comparative efficacy and effectiveness research

\section{Background \& Methodology:}

The Translational Evidence Mechanism explains the dynamic by which the best evidence, from fundamental and proteomics research to clinical trials and systematic reviews, contributes to clinical decision-making, utility, and cost data, and produces individual, not average, patient optimized clinical decisions or choices. This translational process is composed of 3 entities that involve information technology:

Translational Evidence Organization,

Central Database, and

Decision-Making Algorithm, a primary component in constructing, vesting, and maintaining the central database.

The Translational Evidence Organization develops, verifies, maintains, and updates current, best evidence for end-users in their consultations with service providers. The primary purpose of the Translational Evidence Organization is to arbitrate published evidence and, in its absence, identify research that needs to be conducted by organizational affiliates or the research community in producing needed data. A team, composed of basic, translational and professional researchers, vests the central database with evidence and set evidence priorities. Translational researchers also arbitrate published evidence to produce best evidence. Figure 1 illustrates the need of additional, post arbitrators of evidence. These post arbitrators are the providers (clinicians) and consumers (patients).

The Clinicians are concerned with clinical significance and relevance; whether research findings can be applied to individual patients. For dentistry, dentists make judgments that may weight best evidence differently from researchers. Initially, clinical significance of decision data is coordinated with dentists in developing nationally, regionally, or locally relevant best evidence.

The dentists are provided a clinical practice guideline (CPG) with decision and utility best evidence and locally provided cost schedules. Dentists provide assessments of clinical significance of the CPGs based on practice and local factors. These assessments are developed from applying knowledge logically based on concepts learned during training and implicitly in rendering health services based on experience and patient characteristics of well-being. During care delivery, dentists perform assessments, evaluate services needed, and develop plans for treatments and therapies. In providing dental care services, dentists may contribute to the understanding of the "when, where, and how" of knowledge. Dentists' evaluations of clinical relevance is used by translational researchers to reject or modify the clinical practice guidelines or to re-identify and conduct investigations that produce other clinically relevant data. Personal and professional expertise and experiences, values and preferences, and appropriate practices, as well as patient well-being, quality of life issues, and costs weigh heavily on whether best evidence is used in clinical decisions. Thus, dentists are able to predict clinical outcomes in weighing risks against benefits and costs for individual patients. Dentists may also make relevant standards of care in their local practice and for specific patient population characteristics. These clinical validations are necessary to translate research data into clinically useful data for efficacy of patient care.

In the context of effectiveness studies, patients are typically categorized as the consumers of products and services and not the developers or 
guarantors of knowledge. Patients become the "conceptual subject" to which best evidence is applied and quantitative and qualitative outcomes are measured. However, patients may be advocates or adversaries of evidence. Patients may also exert demands on evidence to meet specific, personal needs. They may exert influence on the development and application of knowledge that does not necessarily meet acceptance criteria of researchers and dentists, but serves a personal need. They may also exert pressure to deny the development and application of knowledge that is contrary to their philosophical beliefs.

Even in the profession's best efforts of raising the patients' health literacy by informing them with best evidence and using clinician expertise to communicate individualized, effective treatments, patients ultimately decide if treatment regimens are adhered to or rejected outright. Patient adherence, modification, or rejection of best evidence in treatment scenarios provides translational researchers with its meaning in practice. Translational researchers integrate these evaluations with the knowledge of fundamental biology and proteomic signature in updating clinically relevant data. These updates are processed using decision, Bayesian, and sensitivity analyses within the central database.

The Central Database is the second essential component of the novel and emerging Translational Evidence Mechanism. It represents a repository of evidence, organized and stored utilizing evidence-based research knowledge management software (EBRsoftware), currently in development. EBRsoftware [1] is maintained by information technologists and integrated within the electronic patient chart, and thus is directly integrated with health information technology (HIT) advancements.

Both health information technology and the Internet, as a vehicle for information technology, has become the most cost effective mode of information transfer [2, 3]. It is this inclusive and reciprocal participation of all decision makers that accomplishes improvements in clinical care by means of the translational integration of the patient's fundamental biology, including the outcomes of proteomic studies, with clinical data and observations.

The structure of the central database consists of a primary network. This primary network contains all vested evidence. The network may then be partitioned into subordinate databases. Information is then filtered and segregated per subspecialty information. Subspecialty information is that best evidence used in specific populations, geographic locales: national, regional, specialty, and local secondary networks, specialty procedural treatments, quality assurance, costs, and care delivery including facility or equipment improvements. These subspecialty databases may facilitate access based on frequency of usage.

When uncertainties in patient care arise, the clinician queries the central database through the patient's chart. EBRsoftware administers the query and vests CPG with best decision for efficacy (i.e., satisfaction measures, or trade-offs), and effectiveness (e.g., utility, risk/benefit, and cost data). If preferred, cost data may be converted to practice schedules through the electronic chart.

Once vested, CPG is returned dated and specified as to expiration date of the evidence provided, the functional status (functionally independent, frail, or functionally dependent) of the patient to which the evidence applies, and, if available, the significance (statistical and clinical), utility ranking, and meaning in practice of the evidence. The returned CPG is interactive in that it provides stratified risk scenarios for high, equipoise, or low risk takers that may be accessed and "gamed" through a drop down menu. With the patient's involvement, returned quantitative and qualitative best evidence is organized into decision, utility, and cost data for each possible option/outcome to assist in shared decision-making. If utility rankings, based on the "average patient," are not acceptable to the patient, these may be changed through drop down menus, reflecting personal preferences, values, and beliefs regarding the presented outcomes. Thus, the patient may change, or "game," rankings to compare and test different treatment scenarios in optimizing the clinical decision or provide assessments to compare personal trade-offs between options with the "average patient." The objective of patient "gaming" is to optimize the clinical decision. Accompanying the CPG exhibit is the decision analysis statement. This statement provides a written interpretation or meaning of the treatment scenarios and their calculations.

The Decision-Making Algorithm constitutes the third dynamic component of the Translational Evidence Mechanism. At this stage, the decision analysis of the CPG is done in three articulated steps:

Interpreting for the patient the probability of the outcome;

Multiplying utility data by the probability of the outcome in interpreting the service benefit to the patient; and

Multiplying the probability of the outcome by the treatment cost in determining the economic choice difference between outcomes.

By means of this approach, the analyses outlined above become rollback calculations to determine individual options, which help guide patients in determining their optimal treatment decision.

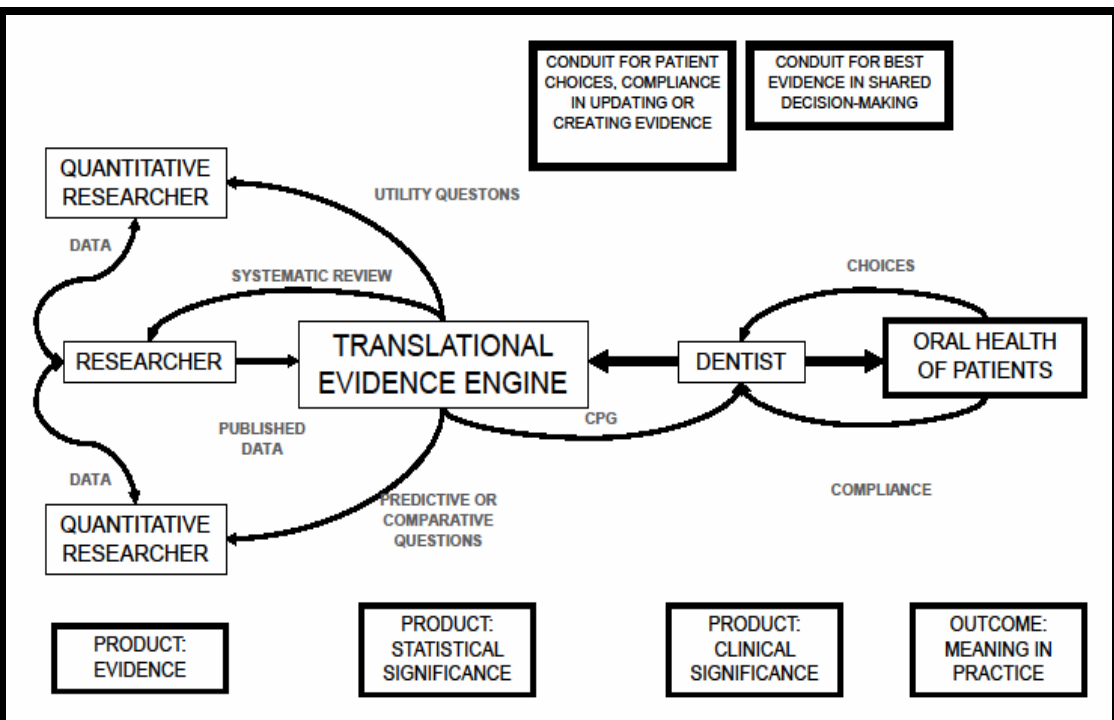

Figure 1: Transforming scientific evidence for shared-decision making in arriving at individualized, consumer choices. 


\section{Bioinformation Volume 5}

\section{Conclusion:}

In brief, the resultant patient analysis and optimal clinical decision is returned to the central database to be retrieved by translational researchers to develop new research questions or modify existing evidence. Having an efficient decision-making process has an additional benefit of involving private practice as a unit in clinical research without disrupting normal patient flow or care. Conversely, clinicians may compare local based practice norms, patient characteristics, and standards to those derived on a regional and national basis.

The Translational Evidence Mechanism is non-disciplinary specific. It is equally successfully applicable and applied to any discipline that services clients or patients, researchers, and policy-makers because it integrates fundamental molecular biology and pathology data obtained from the patient with clinical observation, tests, expertise, as well as the best available evidence generated from research synthesis methodology and meta-analysis. It is predicted that the Translational Evidence Mechanism, which we outline here (Figure 1) will increasingly gain hold among researchers, patients and clinicians alike, because it empowers all involved constituencies and stakeholders in improving the efficacy and the effectiveness of treatment interventions through a systematic scientific process of clinically relevant complex systematic reviews [4] and comparative efficacy and effectiveness research and analysis for practice [5].

\section{Acknowledgements:}

The authors the students and colleagues who contribute to the growth and establishment of the novel emerging field of translational comparative efficacy and effectiveness research and analysis in dental practice

\section{References:}

[1] J Bauer US Patent Application No. 60/757,647, Filed: January 9, 2006.

[2] S Bakken, J. McArthur J. Am. Med. Inform. Assoc. 8: 289 (2001) [PMID: 11320073]

[3] Integrated Healthcare Association. Pay for Performance (P4P) 2008 Results Report. August 2009.

[4] F Chiappelli et al. The Open Dent. J. 4: 48 (2010) [PMID: 20871750]

[5] A Barkhoardarian et al. Pathology Research International (2011) (in press)

Edited by F Chiappelli

Citation: Bauer \& Chiappelli, Bioinformation 5(7): 297-299 (2011)

License statement: This is an open-access article, which permits unrestricted use, distribution, and reproduction in any medium, for non-commercial purposes, provided the original author and source are credited. 